\title{
Analytic and Numerical Solutions of Couette Flow Problem: A Comparative Study
}

\author{
Dhak Bahadur Thapa ${ }^{1}$, Kedar Nath Uprety ${ }^{2}$ \\ ${ }^{I}$ Department of Mathematics, Damak Multiple Campus, Jhapa, Nepal \\ ${ }^{2}$ Central Department of Mathematics, Tribhuvan University, Kathmandu, Nepal \\ Corresponding author:db.thapa12@gmail.com ; kedar021@hotmail.com
}

Received: June 21, 2016

Revised: July 15, 2016

Accepted: July 5, 2016

\begin{abstract}
In this work, an incompressible viscous Couette flow is derived by simplifying the Navier-Stokes equations and the resulting one dimensional linear parabolic partial differential equation is solved numerically employing a second order finite difference Crank-Nicolson scheme. The numerical solution and the exact solution are presented graphically.
\end{abstract}

Key Words: Navier-Stokes Equations, Couette Flow, Finite Difference, CrankNicolson Scheme.

\section{Introduction}

Couette flow [1] is a viscous flow between two parallel plates separated by some vertical distance. The upper plate is moved with velocity $u_{e}$ and the lower plate is kept stationary i.e. its velocity $u=0$. The flow is two dimensional in $x y$ palne. The flow field between the two plates is to be driven exclusively by the shear stress exerted on the fluid by the moving upper plate so that the velocity profile is formed across the flow.

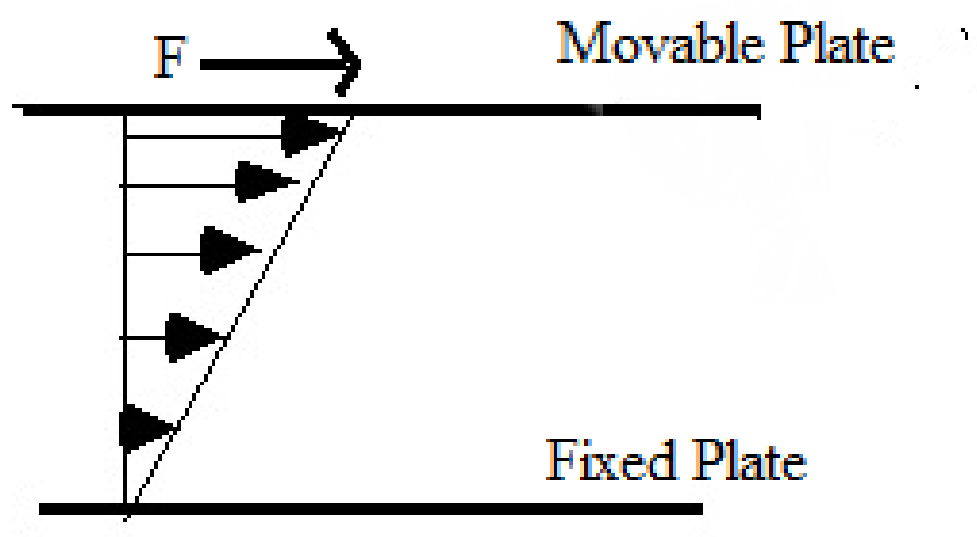

Fig. 1: Flow between two parallel plates 
The assumptions [1] of the Couette Flow problem are as follows:
a. Flow between two parallel plates separated by some vertical distance.
b. Upper plate is moved and the lower plate is kept stationary.
c. Flow is two dimensional in $x y$ palne.
d. Flow extends from $-\infty$ to $+\infty$ in $x$-direction.
e. Flow field variables are independent of $x$.
f. Vertical component of velocity is absent.
g. Streamlines for the Couette flow are straight lines.
h. Pressure gradients in both $x$ and $y$ directions are zero.

\section{Derivation of Governing Equation}

The Couette flow equation [3] is the $x$-momentum equation in $2 D$ given by

$$
\frac{\partial(\rho u)}{\partial t}+\frac{\partial\left(\rho u^{2}\right)}{\partial x}+\frac{\partial(\rho u v)}{\partial y}=-\frac{\partial p}{\partial x}-\frac{\partial \tau_{x x}}{\partial x}-\frac{\partial \tau_{x y}}{\partial y}+\rho f_{x} .
$$

In the absence of body force, this equation for variable viscosity case becomes

$$
\frac{\partial u}{\partial t}+u \frac{\partial u}{\partial x}+v \frac{\partial u}{\partial y}+\frac{1}{\rho} \frac{\partial p}{\partial x}=\frac{\partial}{\partial x}\left(v \frac{\partial u}{\partial x}\right)+\frac{\partial}{\partial y}\left(v \frac{\partial u}{\partial x}\right)
$$

where $\vec{v}=(u, v)$ is the velocity field, $\rho$ is the density and $v$ is the coefficient of viscosity of the fluid. The model for Couette flow extends from $-\infty$ to $+\infty$ in $x$-direction. Since there is no begining or end of this flow, the flow field variables must be independent of $x$. Moreover, the equation of continuity for steady-state flow is

$\frac{\partial(\rho u)}{\partial x}+\frac{\partial(\rho v)}{\partial y}=0$.

Since $\frac{\partial(\rho u)}{\partial x}=0$ for Couette flow, equation (2) reduces to

i.e.

$$
\frac{\partial(\rho v)}{\partial y}=0
$$

At the lower wall, $v=0$ at $y=0$. So, the equation (3) reduces to

$$
\left(\frac{\partial v}{\partial x}\right)_{y=0}=0 \text {. }
$$

Expanding $v$ in Taylor's series about the point $y=0$, we get

$$
v(y)=v(0)+\left(\frac{\partial v}{\partial y}\right)_{y=0}+\left(\frac{\partial^{2} v}{\partial y^{2}}\right)_{y=0} \frac{y^{2}}{2}+\cdots .
$$

At the upper wall, we have

$$
v(D)=v(0)+\left(\frac{\partial v}{\partial y}\right)_{y=0} D+\left(\frac{\partial^{2} v}{\partial y^{2}}\right)_{y=0} \frac{D^{2}}{2}+\cdots .
$$

Since both $v(D)=0$ and $v(0)=0$ as well as $\left(\frac{\partial v}{\partial y}\right)_{y=0}=0$, the only result from equation (6) that makes sense is 


$$
\left(\frac{\partial^{n} v}{\partial x^{n}}\right)_{y=0}=0 \text { for all } \mathrm{n}
$$

This implies that

$$
v=0 \text {. }
$$

everywhere. Thus, there is no vertical velocity in Couette flow. Therefore, the streamlines for the Couette flow are straight lines which runs parallel to each other. Also the $y$-momentum equation is

$$
\rho \frac{D v}{D t}=\frac{\partial p}{\partial y}+\frac{\partial \tau_{x y}}{\partial x}+\frac{\partial \tau_{y y}}{\partial y}+\rho f_{x}
$$

For the Couette flow with no body forces, it reduces to

$$
-\frac{\partial p}{\partial y}+\frac{\partial \tau_{y y}}{\partial y}=0
$$

But, we have

$$
\tau_{y y}=\lambda\left(\frac{\partial u}{\partial x}+\frac{\partial v}{\partial y}\right)+2 \mu \frac{\partial v}{\partial y}=0 .
$$

With this equation in hand, eqution (8) becomes,

$$
\frac{\partial p}{\partial y}=0 \text {. }
$$

Thus, there are no pressure gradient terms in both $\mathrm{x}$ and $\mathrm{y}$ directions. Therefore, for two dimensional steady-state Couette-Flow with no body force, the x-momentum equation (1) becomes

$$
\rho u \frac{\partial u}{\partial x}+\rho v \frac{\partial u}{\partial y}=-\frac{\partial p}{\partial x}+\frac{\partial \tau_{x x}}{\partial x}+\frac{\partial \tau_{y x}}{\partial y} .
$$

But for the Couette flow, we have

$$
\tau_{x x}=\lambda\left(\frac{\partial u}{\partial x}+\frac{\partial v}{\partial y}\right)+2 \mu \frac{\partial v}{\partial x}=0 .
$$

and

$$
\tau_{y x}=\lambda\left(\frac{\partial v}{\partial x}+\frac{\partial u}{\partial y}\right)=\mu \frac{\partial u}{\partial y} .
$$

Substituting these values in (10), we get

$$
\frac{\partial}{\partial y}\left(\mu \frac{\partial u}{\partial y}\right)=0 \text {. }
$$

If the flow is incompressible with constant temperature, this equation reduces to

$$
\frac{\partial^{2} u}{\partial y^{2}}=0
$$

Similarly, the $x$-momentum equation for unsteady, incompressible Couette flow is

$$
\rho \frac{\partial u}{\partial t}=\mu \frac{\partial^{2} u}{\partial y^{2}}
$$

It is our governing equation, the Couette flow for incompressible viscous fluid which is a parabolic PDE. 


\section{Analytic Solution}

The governing equation for unsteady incompressible viscous Couette flow is

$$
\rho \frac{\partial u}{\partial t}=\mu \frac{\partial^{2} u}{\partial y^{2}}
$$

The equation in non-dimensional form is

$$
\begin{aligned}
& \rho \frac{\partial\left(\frac{u^{\prime}}{u_{e}}\right)}{\partial\left(\frac{t^{\prime}}{D / u_{e}}\right)}\left(\frac{u_{e}{ }^{2}}{D}\right)=\mu \frac{\partial^{2}\left(\frac{u^{\prime}}{u_{e}}\right)}{\partial\left(\frac{y^{\prime}}{D}\right)^{2}}\left(\frac{u_{e}}{D^{2}}\right) . \\
& \frac{\partial u^{\prime}}{\partial t^{\prime}}=\frac{\mu}{\rho u_{e} D} \frac{\partial^{2} u^{\prime}}{\partial y^{\prime 2}} .
\end{aligned}
$$

Since

$$
\frac{\mu}{\rho u_{e} D}=\frac{1}{R e_{D}}
$$

where $R e_{D}$ is the Reynolds number based on the height $\mathrm{D}$ between the two plates. Thus, equation (13) becomes

$$
\frac{\partial u^{\prime}}{\partial t^{\prime}}=\frac{1}{R e_{D}} \frac{\partial^{2} u^{\prime}}{\partial y^{\prime 2}}
$$

Equation (14) is the PDE for which we require exact and numerical solutions. First, we write the equation (14) in the form

$$
\frac{\partial u}{\partial t}=\frac{1}{R e_{D}} \frac{\partial^{2} u}{\partial y^{2}}
$$

where $\mathrm{u}, \mathrm{t}$ and $\mathrm{y}$ are identical with that of dashes in equation (14). To solve the equation (15) analytically, let us consider it in the form,

$$
u_{t}=\kappa u_{x x}, 0<x<l, t>0,
$$

where $\kappa=\frac{1}{R e_{D}}$. It is a one dimensional heat equation. Suppose $u(x, t)$ be the solution of equation (16) representing the temperature distribution in a homogeneous rod of length 1 and let it satisfies the boundary and initial conditions

$$
\left\{\begin{array}{l}
u(0, t)=0=u(l, t), t \geq 0 \\
u(x, 0)=f(x), 0 \leq x \leq l .
\end{array}\right.
$$

Let us assume the seperable solution of the equation (16) in the form

$$
u(x, t)=X(x) T(t) \neq 0 .
$$

Then the equation (16) takes the form

$$
\frac{1}{X} \frac{d^{2} X}{d x^{2}}=\frac{1}{\kappa T} \frac{d T}{d t} .
$$

Since the left hand side depends only on $x$ and right side is the function of $t$ only, equation (19) is considered true only when we have

$$
\frac{1}{X} \frac{d^{2} X}{d x^{2}}=\frac{1}{\kappa T} \frac{d T}{d t}=\lambda
$$

This generates two ODE's namely 
and

$$
\begin{aligned}
& \frac{d^{2} X}{d x^{2}}=\lambda X, \\
& \frac{d T}{d t}=\lambda \kappa T .
\end{aligned}
$$

It is easy to find the final form of the solution [6] of the equations in (20) which is given by

$$
u(x, t)=\sum_{n=1}^{\infty}\left(\frac{2}{l} \int_{0}^{l} f(\xi) \sin \left(\frac{n \pi \xi}{l}\right) d \xi\right) \exp \left(\frac{n^{2} \pi^{2} \kappa t}{l^{2}}\right) \sin \left(\frac{n \pi x}{l}\right) .
$$

Physically, the temperature distribution decays exponentially with time $t$. This shows a striking contrast to the wave equation whose solution oscillates in time t. The time scale of decay for the $n^{\text {th }}$ mode is given by

$$
T_{d}=\frac{1}{\kappa}\left(\frac{l}{n \pi}\right)^{2},
$$

which is directly proportional to $l^{2}$ and inversly proportional to the thermal conductivity $\kappa$.

\section{Numerical Solution}

Here we make use of second order finite difference Crank-Nicolson Scheme [7] for the numerical approximation of the equation, because it is an implicit scheme which is unconditionally stable and hence is convergent. So the approximation of the solutions by this scheme is better than any other explicit schemes. If $v_{n}$ denotes a smooth function approximating the function $u(x, t)$ in the Couette flow equation

$$
\frac{\partial u}{\partial t}=\frac{1}{R e_{D}} \frac{\partial^{2} u}{\partial y^{2}}
$$

then the Crank-Nicolson scheme for this equation is

$$
\begin{gathered}
\frac{v_{j}^{n+1}-v_{j}^{n}}{\Delta t}=\frac{1}{R e_{D}} \frac{\left[\frac{1}{2}\left(v_{j+1}^{n+1}+v_{j+1}^{n}\right)-\left(v_{j}^{n+1}+v_{j}^{n}\right)+\frac{1}{2}\left(v_{j-1}^{n+1}+v_{j-1}^{n}\right)\right]}{(\Delta y)^{2}} . \\
\text { i.e., } \quad\left(-\frac{\Delta \mathrm{t}}{2(\Delta y)^{2} R e_{D}}\right) v_{j-1}^{n+1}+\left(1+\frac{\Delta t}{(\Delta y)^{2} R e_{D}}\right) v_{j}^{n+1}+\left(-\frac{\Delta \mathrm{t}}{2(\Delta y)^{2} R e_{D}}\right) v_{j+1}^{n+1}= \\
\left(1-\frac{\Delta t}{(\Delta y)^{2} R e_{D}}\right) v_{j}^{n}+\frac{\Delta \mathrm{t}}{2(\Delta y)^{2} R e_{D}}\left(v_{j+1}^{n}+\right.
\end{gathered}
$$

$\left.v_{j-1}^{n}\right)$.

This is in the form

$$
A v_{j-1}^{n+1}+B v_{j}^{n+1}+A v_{j+1}^{n+1}=K_{j} .
$$

where,

and

$$
\begin{aligned}
& A=-\frac{\Delta \mathrm{t}}{2(\Delta y)^{2} R e_{D}}, \\
& B=1+\frac{\Delta \mathrm{t}}{(\Delta y)^{2} R e_{D}}, \\
& K_{j}=\left(1-\frac{\Delta t}{(\Delta y)^{2} R e_{D}}\right) v_{j}^{n}+\frac{\Delta \mathrm{t}}{2(\Delta y)^{2} R e_{D}}\left(v_{j+1}^{n}+v_{j-1}^{n}\right) .
\end{aligned}
$$


Now, we solve equation (21) on a grid such that the vertical distance D between the plates is divided into $\mathrm{N}$ equal parts by administrating $(\mathrm{N}+1)$ grid points over the vertical height $\mathrm{D}$,

$$
\text { i.e., } \quad \Delta y=\frac{D}{N} \text {. }
$$

We impose the boundary conditions non-dimensional velocity $v$ as

$$
v_{1}=0
$$

and

$$
v_{N+1}=1 \text {. }
$$

As equation (21) represents a system of ( $\mathrm{N}-1)$ equations in $(\mathrm{N}-1)$ unknowns namely, $v_{2}, v_{3}, \ldots \ldots, v_{N-1}$, the first equation of the system is

$$
A v_{1}^{n+1}+B v_{2}^{n+1}+A v_{3}^{n+1}=K_{2}
$$

But $v_{1}=0$, we have

$$
B v_{2}^{n+1}+A v_{3}^{n+1}=K_{2} .
$$

The last equation of the system (21) is

$$
A v_{N-1}^{n+1}+B v_{N}^{n+1}+A v_{N+1}^{n+1}=K_{N} .
$$

But $v_{n+1}=1$, we have

$$
A v_{N-1}^{n+1}+B v_{N}^{n+1}=K_{N}-A v_{e} .
$$

With the equations (28) and (29), the system of equations (21) can be expressed in matrix form as

$$
\left(\begin{array}{cccccccc}
B & A & 0 & 0 & \cdots & 0 & 0 & 0 \\
A & B & A & 0 & \cdots & 0 & 0 & 0 \\
0 & A & B & A & \cdots & 0 & 0 & 0 \\
0 & 0 & A & B & \cdots & 0 & 0 & 0 \\
0 & 0 & 0 & A & \cdots & 0 & 0 & 0 \\
\cdots & \cdots & \cdots & \cdots & \ddots & \cdots & \cdots & \cdots \\
0 & 0 & 0 & 0 & \cdots & A & B & A \\
0 & 0 & 0 & 0 & \cdots & 0 & A & B
\end{array}\right)\left(\begin{array}{c}
v_{2}^{n+1} \\
v_{3}^{n+1} \\
v_{4}^{n+1} \\
\vdots \\
v_{N-1}^{n+1} \\
v_{N}^{n+1}
\end{array}\right)=\left(\begin{array}{c}
K_{2} \\
K_{3} \\
K_{4} \\
\vdots \\
K_{N-1} \\
K_{N}-A v_{e}
\end{array}\right)
$$

It is a tridiagonal matrix and can be solved by using Thomas algorithm. By the algorithm, we find the solution for $v_{2}^{n+1}, v_{3}^{n+1}, \ldots \ldots ., v_{N-1}^{n+1}$, the velocities at the $(n+1)^{t h}$ time level. Then, the whole process is repeated for a number of time step until the velocity profile converges to a steady state.

\section{The Setup}

For the specific solution, We choose $\mathrm{N}+1$ grid points along vertical, so that the space size is

$$
\Delta y=\frac{1}{N} .
$$

We choose the initial conditions as

$$
v_{i}=0 \text { for } i=1,2, \ldots \ldots, N \text { at } t=0 .
$$

and the time step size $\Delta t$ so as to satisfy the CFL condition,

$$
\Delta t \leq \frac{1}{2} R e_{D}(\Delta y)^{2} .
$$


But we have the Crank Nicolson scheme in the present context which is unconditionally stable, we are free to choose time step size $\Delta t$.

Example: Consider a PDE $\quad u_{t}=a u_{x x}$ with boundary and initial conditions

and

$$
\begin{aligned}
& u(0, t)=u(1, t)=0, \\
& u(x, 0)=f(x) .
\end{aligned}
$$

The exact solution [5] of the PDE is $\quad u(x, t)=\sum_{n=1}^{\infty} A_{n} e^{-a n^{2} \pi^{2} t} \sin (n \pi x)$.

Taking $f(x)=2 \sin (2 \pi x)$. For $R e_{D}=10$ i.e. $a=\frac{1}{10}$, the graphical solution of the equation is obtained from the Matlab implementation which is a $3 \mathrm{D}$ surface as shown in the following graph. It is clear from the graph that the solutions behave as a sinusoidal function at the lower time level and as the time level increases, it becomes more and more consistent and finally all the solutions appear to be coplanar so that the solution set appear a plane.

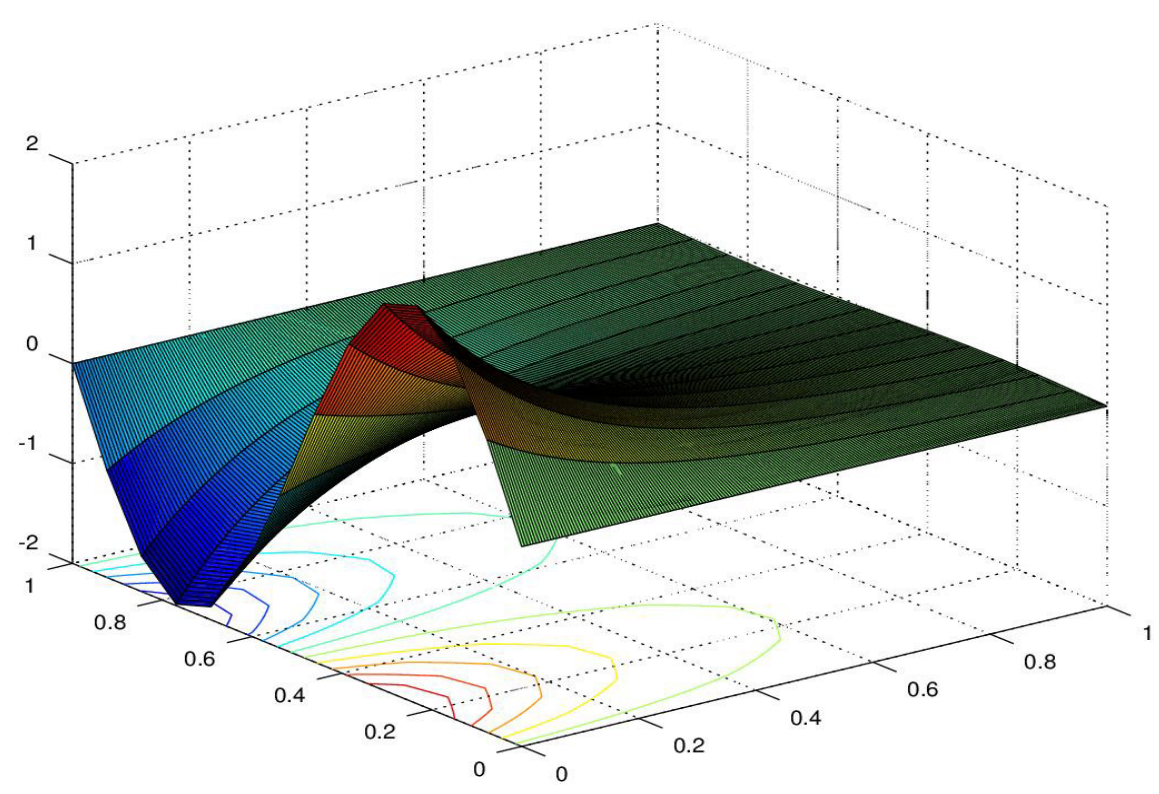

Fig. 2: Velocity profile (numerical solutions)

The graph of the initial condition with Matlab implementation appears as below which is exactly similar to the velocity profile. This shows that the initial conditions also satisfy the velocity profile. 


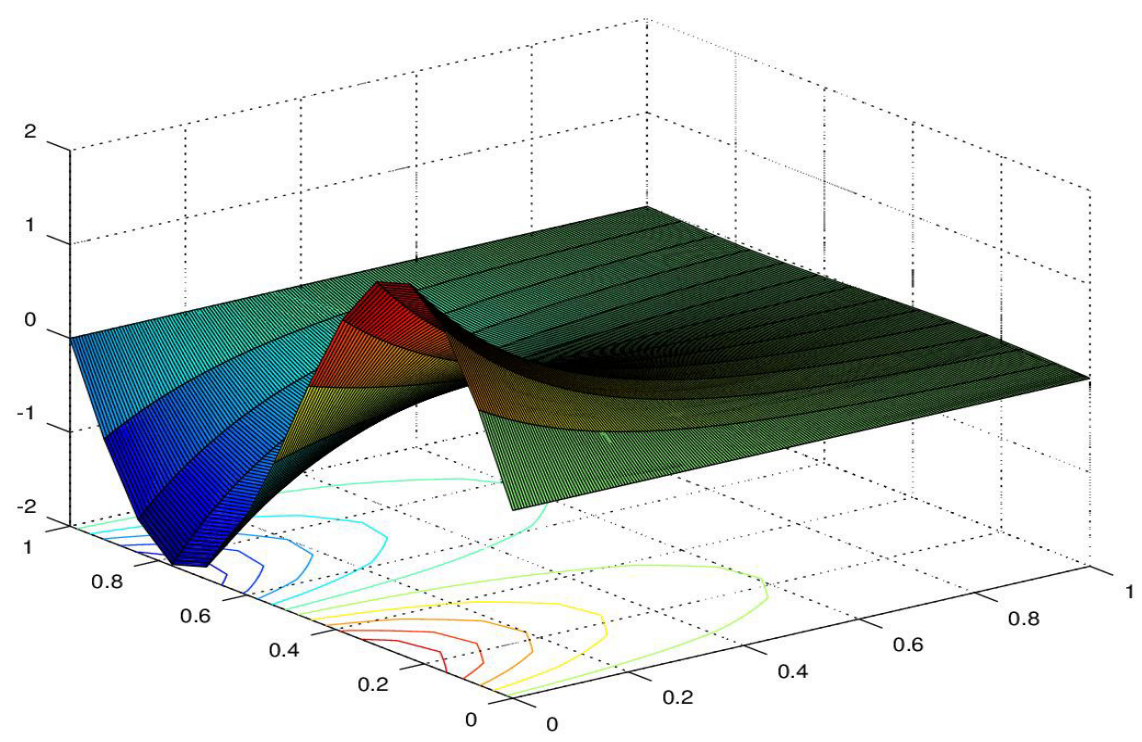

Fig. 3: Graph of Initial Conditions.

We also can observe the various graphs of the solutions by changing time step size $\Delta t$, space step size $\Delta x$ and the Reynolds numbers $R e_{D}$. While changing the values, we should note that the CFL condition should be satisfied. Otherwise, the scheme will be unstable and so the graphical output will not be appropriate.

The exact solution is presented below graphically with the help of Matlab implementation.

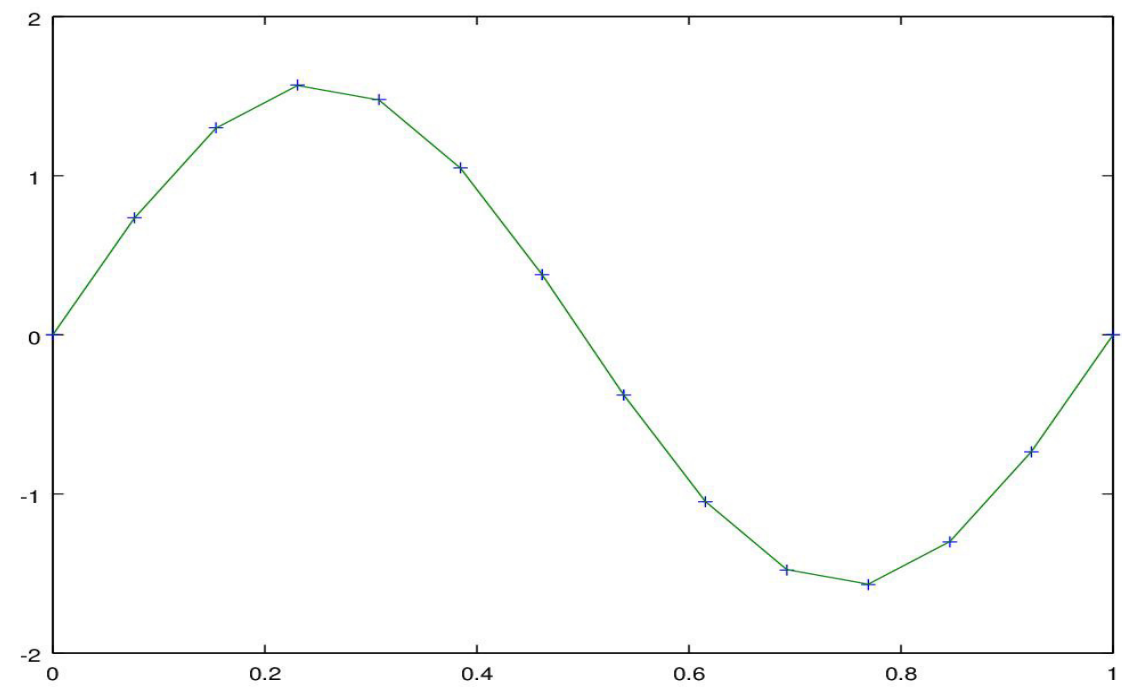

Fig. 4: Graphical representation of exact solution 


\section{Result and Conclusion}

Mathematics is an elegant and precise subject. However, when numerical answers are required we sometimes need to rely on approximate methods to predict answers. There are many problems which simply do not have analytical solutions or may be beyond our current state of knowledge. There are also many problems which are too long (or tedious) to solve by hand. When such problems arise, we can use numerical analysis to reduce the problem to one involving a finite number of unknowns and use a computer to solve the resulting equations.

From this study, we observed that it is easy to find the exact solution of the Couette Flow problem. Since the numerical method gives only the numerical solutions, it mayn't be appropriate in the case when the exact solutions are available. The use of the finite difference method in this paper (Couette flow problem) is to verify its use in complex problems where the existing knowledge of mathematics is not sufficient for their solutions and it is proved from this work that the numerical scheme (Crank Nicolson) is the best approximation to the solutions of Couette flow problem. Therefore, one can say on the basis of this experiment that all convergent numerical schemes can approximate the exact solutions with desired degree of accuracy. Because of such successes of the numerical method, it is proved to be a good and applicable mathematical tool to deal with any sort of real life problems.

\section{References}

[1] Anderson Jr. JD (1995), Computational Fluid Dynamics. McGraw-Hill, New York.

[2] Bhattacharyya B (2009), Fluid Dynamics. New Central Book Agency, New Delhi.

[3] Debenath L (2005), Nonlinear Partial Differential Equations for Scientists and Engineers. Brikhauiser, Boston.

[4] Sauer T (2006), Numerical Analysis. Pearson Education, Inc., Boston.

[5] Sengupta T (2004), Computational Fluid Dynamics. University press, Hydarabad, India.

[6] Strass W (2007), Partial Differential Equations. John Weiiley and Sons ltd.

[7] Strikwerda JC (2004), Finite Difference Schemes and Partial Differential Equations. SIAM, Philadelphia, USA. 